\title{
A prendiendo el
}

\section{arte marcial indio}

del kalarippayattu:

ecologías inciertas de

género, clase, cultura y

etnicidad

Sara K. Schneider

National-Louis University (EE.UU.).

Es traducción del artículo Learning India's Martial Art of Kalarippayattu: Unsettled Ecologies of Gender, Class, Culture, and Ethnicity, publicado en el Journal of Asian

Martial Arts, volumen 19, número 3 (46-63), 2010

\section{Resumen}

Sobre la base de la experiencia de campo de la autora en el estudio del arte marcial tradicional del sur de la India del kalarippayattu, este artículo examina las complejidades de la comunicación, incluyendo la utilización del tacto como herramienta educativa, en una relación intercultural profesor-alumno, y resalta cómo el género, la cultura y la clase tienen un impacto en el aprendizaje de esta forma de arte encarnado 1.

Palabras clave: Relaciones interculturales, relación profesor-alumno, gurú, discípulo, contacto físico, contacto verbal.

\section{Learning India's M artial Art of Kalarippayattu: Unsettled Ecologies of Gender,} Class, Culture, and Ethnicity

Abstract: Rooted in the author's field experience studying the traditional South Indian martial art kalarippayattu, this article examines the complexities of communication, including the use of touch as a teaching tool, in an intercultural teacher-student relationship, and surfaces how gender, culture, and class impacted learning in this embodied art form.

Key words: Intercultural relationships, teacher-student relationship, guru, disciple, physical contact, verbal contact.

${ }^{1}$ N.T: Términos tales como "embodied" o "embodiment" son de uso corriente en la literatura científica angloparlante. Si bien no tienen una traducción al castellano, hemos decidido traducirlos como encarnado y encarnación, términos que ya existen en nuestro idioma y que evitan neologismos tales como encorporado o encorporación ("body": cuerpo). 
Aprendendo a arte marcial índia de kalarippayattu: ecologias incertas de género, classe, cultura e etnicidade

Resumo: Com base na experiência de campo da autora no estudo da arte marcial tradicional da índia de kalarippayattu, este artigo examina as complexidades da comunicação, incluindo a utilização do tacto como ferramenta educativa, numa relação inter-cultural professor-aluno, e destaca como o género, a cultura e a classe têm um enorme impacto na aprendizagem desta forma "vermelha" de arte.

Palavras-chave: Relações inter-culturais, relações professor-aluno, guru, discípulo, contacto físico, contacto verbal.

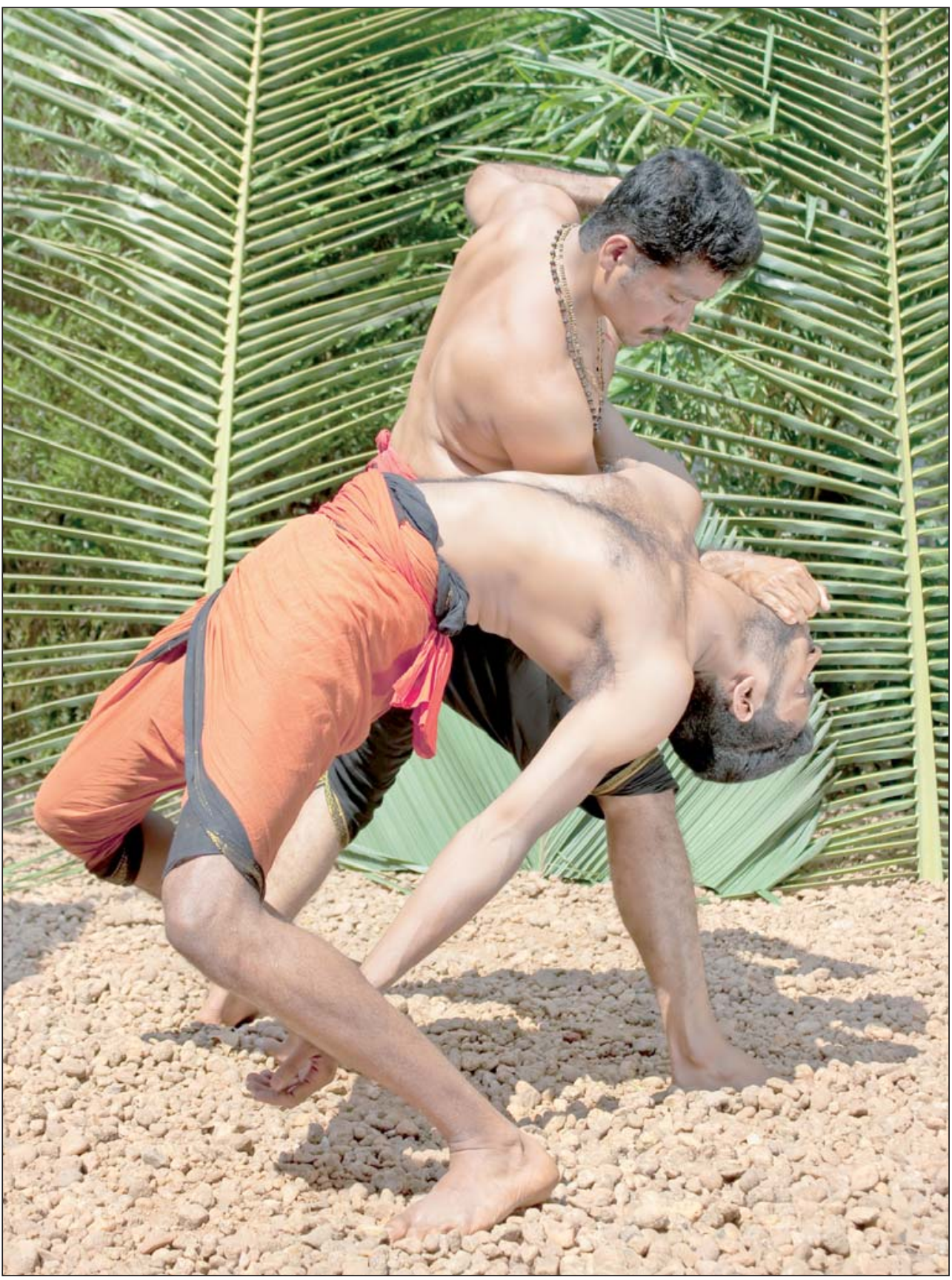

Ilustración cortesía de www.dreamstime.com 


\section{A prendiendo el}

arte marcial indio

del kalarippayattu:

ecologías inciertas de

género, clase, cultura y

etnicidad

Sara K. Schneider

National-Louis University (EE.UU.).

\section{Introducción}

El centro de interés del presente artículo es la relación intercultural, intergénero y de gurú-estudiante en el kalarippayattu, un arte marcial autóctono del sur de la India. En él considero que tanto el aprendizaje como la relación profesoralumno están influidos por las expectativas del que aprende y del que enseña, así como por sus creencias respecto a lo que representa un comportamiento de género, cultura y de clase adecuado. Los mismos medios de enseñanza en esa forma psicofísica -atención; discurso y silencio; gestos, tacto y quietud- también implican marcos culturales en contraste.

Centrado en la relevancia de dos episodios que ocurrieron en 2002 durante mi trabajo de campo y mi observación participante en Calicut, Kerala, India, este artículo saca a la superficie tanto las limitaciones como los logros de acometer un estudio en otros escenarios culturales, así como los anhelos y frustraciones inherentes al trabajo de campo. Un ejemplo etnográfico específico recalca las complejas ecologías del aprendizaje, donde, aparentemente, el contenido se entrecruza con las relaciones profesor-alumno y con las complejidades de género, clase, cultura y etnicidad.

Los datos provienen de las notas de campo, grabaciones y fotografías que tomé durante dos meses de trabajo de campo en la India en el periodo de mayoseptiembre de 2002, así como de las entrevistas que llevé a cabo con el kalarippayattu gurukal (profesor/maestro) T. Sudhakaran, su hija adolescente y estudiante, Archana, y grupos de sus estudiantes masculinos y femeninos de edad escolar. Tal narración de las complejidades interculturales, de la enseñanza y aprendizaje corporal tiende un puente hacia la literatura sobre la relación profesor-estudiante, 
la educación internacional y la antropología del cuerpo. Además resalta la centralidad de la encarnación en los procesos de enseñanza y aprendizaje en el contexto internacional y estadounidense (Classen, 1993; Cooks \& LeBesco, 2006; Freedman \& Holmes, 2003; Geurts, 2002; Lave, 1977; Light, 2001; N ess, 1992).

Con sus raíces remontándose al menos al $\mathrm{s}$. XII, el kalarippayattu es practicado tradicionalmente por la casta $\mathrm{N}$ ayar, si bien también lo practicaban miembros de otros grupos del sur de la India. En malayalam, el lenguaje de Kerala, su nombre significa ejercicios (payattu) realizados en un lugar de práctica (kalari). Revivido en la década de 1920 como parte de la resistencia de Kerala al mandato colonial británico, el kalarippayattu está íntimamente asociado a las imágenes Malayali sobre la virilidad (Zarrilli, 2000). Los chicos que entrevisté en el C.V.N Kalari en Calicut asociaban la práctica con "hacerse fuertes y sanos", con la excelencia en el deporte y con la mejora de la concentración (10 estudiantes masculinos del C.V.N Kalari, comunicación personal, septiembre 2002). Para aquellos que asocian las artes marciales con posturas erguidas como base para ejecutar golpes angulares y secos con las extremidades, una primera mirada al kalaripayyattu puede resultar sorprendente. Sus profundas y anchas sentadillas preparatorias, sus posturas inspiradas en animales y las armas de combate engoladas, con espectaculares saltos y giros, ofrecen una imagen que contrasta con muchas de las artes marciales occidentales y orientales con las que podemos estar más familiarizados.

Las dificultades de la experiencia emergían en parte de mi entrada, mi pertenencia (en cierto grado) y mi marcha de comunidades de aprendizaje de artes marciales como una persona que encarna las ambigüedades de un estatus alto y bajo. Por un lado, era una académica occidental de clase media-alta, frecuentemente tratada (como son tratados los euro-americanos y la gente con buena educación en general) con lo que sentía que era una exagerada deferencia, como si fuera de igual o superior estatus a muchos indios en esta sociedad profundamente jerárquica. (Ozella \& Osella, 1998). Existían también valencias de bajo estatus activamente vinculadas a mí como, después de todo, una mujer soltera, sin hijos en una edad reproductiva, en una cultura que ve a la esposa y a la madre como los roles principales de las mujeres. Estaba eligiendo estudiar un arte marcial que, aunque atrae a chicas jóvenes a su estudio, tiene relativamente pocas mujeres practicantes adultas, y mucho menos chicas

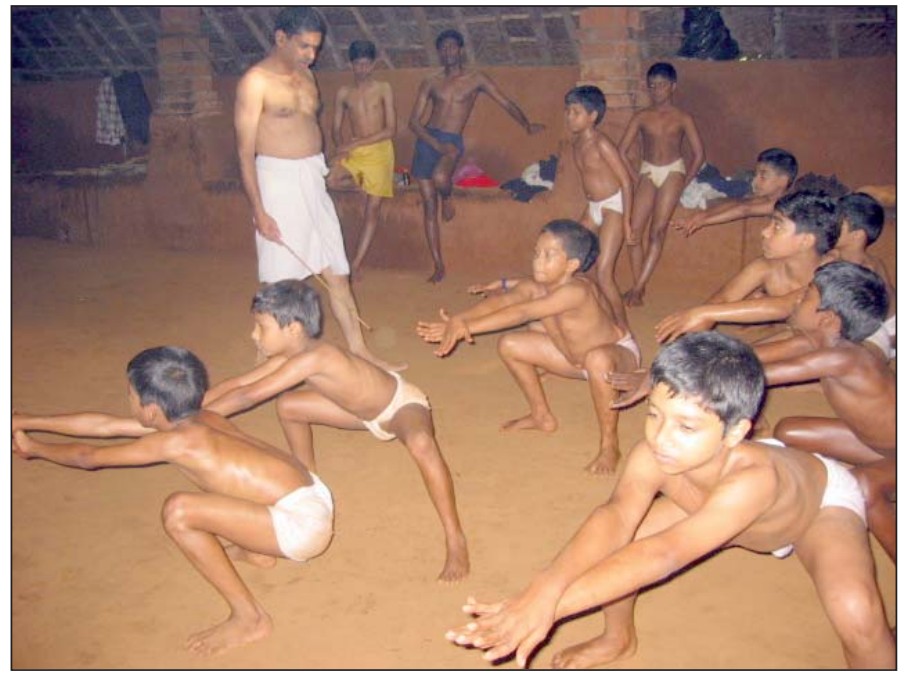
que practiquen después de la pubertad. Aunque continué preguntando bastante en el transcurso y después de las lecciones prácticas, transitando entre una académica implicada verbal y visualmente hacia una estudiante encarnada, sufrí un cambio palpable de estatus, uno que me dejó perpleja en ese momento y que más tarde me reveló una mayor comprensión sobre el papel del tacto y del habla en un sujeto de aprendizaje encarnado. 


\section{El papel del gurú en la transmisión del conocimiento en el kalarippayattu}

En inglés americano, el término "gurú" circula como lo hacen otros términos comerciales como "Kleenex", "Xeros" y "Google". Para los occidentales, el término puede referirse al practicante líder 0 al que marca tendencias, incluso si esa persona no enseña directamente; o puede utilizarse de forma despectiva para designar a un experto con popularidad en la actualidad, aquel al que acuden las emisoras de radio para tener audiencia. En contraste, en la India la figura del gurú se define a través de la relación y de una responsabilidad sagrada. Originariamente, el gurú era un profesor de los textos sagrados védicos y él (porque ciertamente era "él") tenía que ser un miembro de la casta superior de la India, un Bramán; el término gurú podía aplicarse también a profesores de artes y oficios tradicionales (Kale, 1970). Los discípulos del gurú, que podían provenir de cualquier parte de la sociedad india, pasaban su adolescencia viviendo con su familia y recibiendo la tradición de forma oral. Las enseñanzas del gurú eran sacerdotales, realizadas como regalo a sus estudiantes; tan sólo era recompensado al final de los años que pasaban juntos con lo que cada estudiante decidía entregarle.

Con el ascenso del movimiento bhakti en el hinduismo, y su desarrollo a lo largo del período medieval indio, así como la ascendencia del budismo y del jainismo en la India, la noción de gurú como figura de inspiración -incluso sin ser una figura particularmente culta- empezó a emerger. En vez de esperar a que mostrara su valía por su conocimiento, los estudiantes del gurú lo investían con fe. Una forma de adoración del gurú, o gurú yoga, dictaminaba que el discípulo se sometía sin cuestionar, como forma de auto-rendición 0 auto-trascendencia a todo aquello que el gurú pudiera pedir.

Es quizá esta sumisión incuestionada incluida en el término gurú lo que hace que a los occidentales se les pongan los pelos de punta; la aceptación acrítica de alguien como experto puede rallar en lo cultual, en la rendición de culto. Desde esta perspectiva no es menos sospechoso que alguien acepte obediencia ciega a otro. Incluso en la India la visión del gurú como incuestionable erosionó de algún modo el mandato colonial británico, donde los indios experimentaban un tipo muy diferente de profesor, uno enmarañado en una red burocrática cuya última autoridad era el gobierno británico.

Cuando fui a la India, estaba ya de algún modo familiarizada con la figura del gurú por mis dos años de estudio de yoga. En los ashrams estadounidenses (retiros religiosos) he visto discípulos que están de tal modo en manos de la autoridad suprema del gurú que este ha llegado a elegir para ellos un compañero adecuado de matrimonio. Como práctica común en el kalarippayattu, los estudiantes de mi profesor lo llamaban por el plural gurukkal, indicando su representación y encarnación de una larga línea de profesores en su tradición,
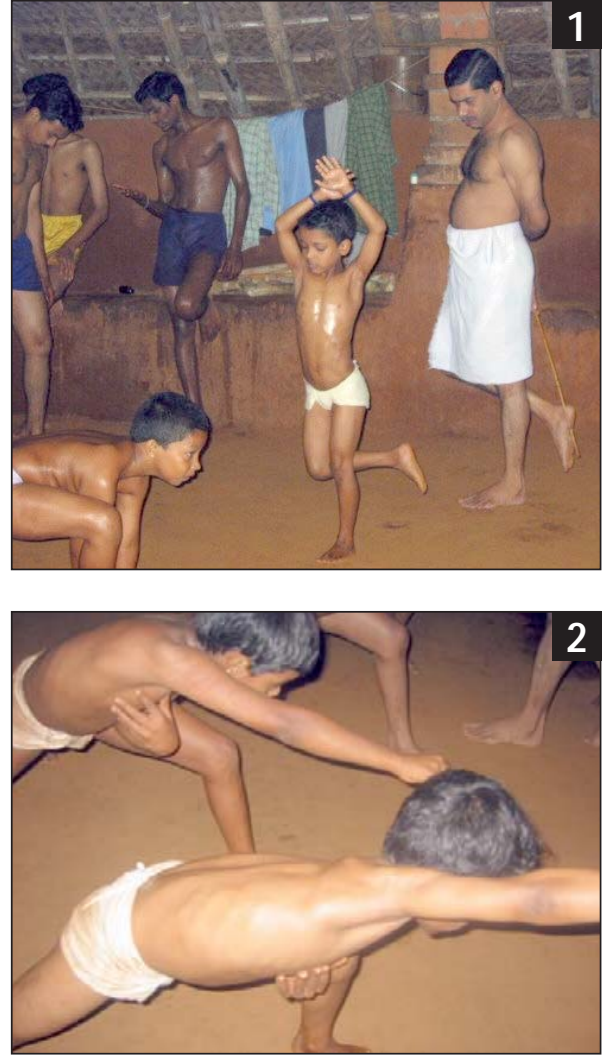

1) El maestro T. Sudhakaran Imitando LOS MOVIMIENTOS DE SU ESTUDIANTE MÁS joven en un Kalari de Calicut, Kerala, INDIA.

2) Parte de la secuencia coreografiada realizada a lo largo del kalari.

3) Práctica concentrada.

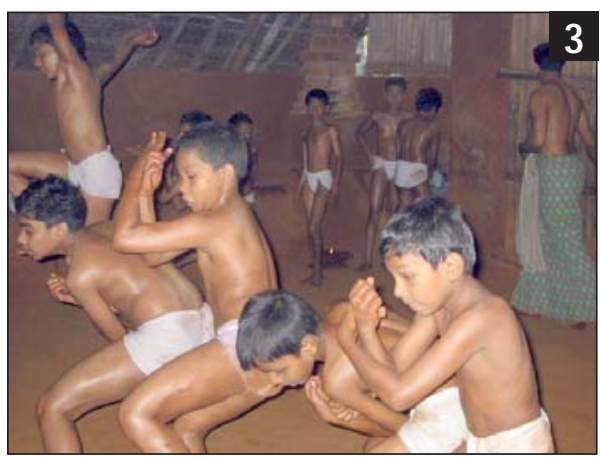




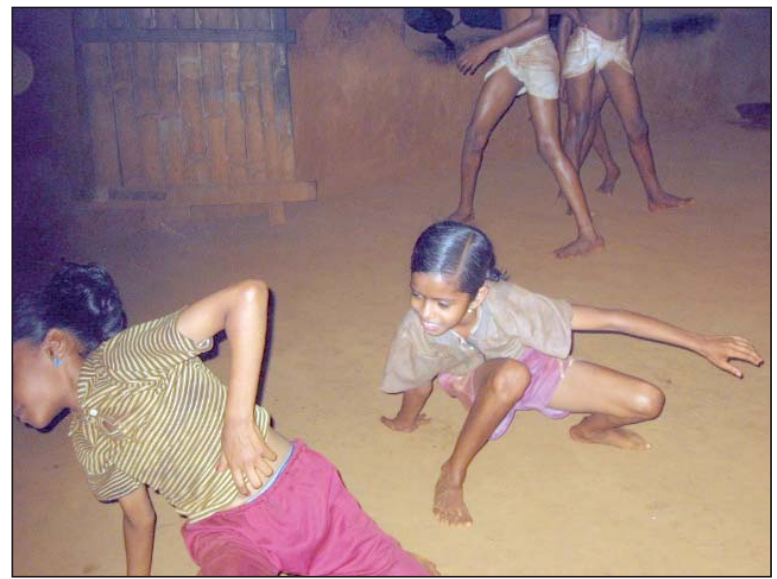

entre los cuales aparece como cima y culminación (Zarrilli, 2000: 301).

Aunque estuve algún tiempo observando la práctica de las artes marciales del kalari del sur con las que el experto occidental, Dr. Phillip B. Zarrilli, está más asociado, no obstante quería llevar a cabo un estudio en una aldea tradicional y remonté la costa para pasar la mayor parte del tiempo en el kalari del norte de Kerala. Durante mi primer viaje a Calicut (Kozhikode), la tercera ciudad más grande de Kerala, fotografié y grabé el trabajo de Sudhakaran con sus estudiantes, observé a los jóvenes en su entrenamiento y su práctica, y me senté en los ensayos y demostraciones del grupo de representaciones de kalarippayattu, que

LAS CHICAS MÁS MAYORES PRACTICANDO EN EL KALARI, OTRA APARTE DE LA HIJA DEL GURÚ, DE 14 AÑOS. hacía espectáculos para turistas hospedados en hoteles de lujo. Sorprendida por el uso de los métodos de enseñanza corporal de ese gurú educado y viajado, escribí en mis notas de campo de mayo de 2002: "El gurukkal enseña mediante diversos métodos. Uno de los más interesantes... es golpear a sus estudiantes con un palo, a veces con dureza. Considera que es un modo de ayudar a los niños a aprender/ recordar. Por supuesto utiliza sus nombres cuando les corrige".

Cuando la amenaza de guerra nuclear con Pakistán surgió en junio de 2002, los extranjeros fueron evacuados del sur de la India. Cuando volví a Kerala en agosto, supe que estudiar con Sudhakaran supondría alterar mi relación con él. Yo había asumido ya profundamente dos roles respecto a él, como investigadora y como posible directora de escenografía, ya que estaba interesada en la creación de una producción más elaborada que mostrara los puntos fuertes de este arte marcial a audiencias nacionales e internacionales. A esos papeles estaba añadiendo el de estudiante 0, como decía Sudhakaran, discípulo, adoptando las costumbres de su kalari. Además, para poder realizar las prácticas de la mañana y de la tarde, me trasladaría desde un hotel de la ciudad de Calicut a las afueras de la ciudad, donde estaba el kalari, alquilando una habitación de su hermano y cuñada, vecinos de Sudhakaran en el complejo familiar.

Después de que Sudhakaran me aceptara como su estudiante en septiembre, luché enconadamente para abandonar mi estatus académico con él. Noté que yo misma me resistía, con todo mi compromiso como estadounidense con el igualitarismo, llamándole con el honorífico gurukkal, que era para mi más adecuado que seguir llamándole por su nombre; asocié el término con una adoración incuestionada que, de nuevo como estadounidense, rehusaba. Tal y como escribí de forma agitada en mis notas de campo,

Si fuera totalmente un gurú para mí -si aceptara esa relación como indiscutible- entonces querría su aprobación totalmente. Querría saber lo que podría hacer mejor como persona. Pero hay una parte de mí que quiere que sigamos siendo colegas de un modo muy informal (que no quiere convertirse en alumna), que quiere criticar sus opiniones despectivamente, o considerar que su propuesta es más primitiva que la mía (sin embargo, ¿qué tiene de primitivo una lameculos???). 
Etnógrafos contemporáneos como Behar (1996) han escrito con sensibilidad sobre las dificultades éticas, relacionales y de procedimiento en sus experiencias de campo. Tras sus explicaciones introductorias de cómo establecen relación con sus sujetos, en las que se encuentra con aspectos inesperados y en las que lleva a cabo prejuiciadas decisiones éticas y prácticas en el transcurso de su recogida de datos y de la escritura de sus estudios, yace su evidente deseo de ayudar a sus audiencias a comprender la metodología y los filtros que han moldeado sus datos. Como lectores, debemos entender que lo que "obtuvieron" era en gran parte lo que ellos, como investigadores indefectiblemente ligados a su propia cultura, eran capaces de "obtener" -de ver, de oír, de comprender-. No existen garantías de que lo que los etnógrafos observan desde fuera es lo que los datos significan en su contexto; en parte, la esperanza del etnógrafo es que, cuando revela al lector sus sesgos, predisposiciones e historia, este lector sea capaz por sí mismo de obtener a través de unas interpretaciones necesariamente parciales una visión más clara de los datos.

Compartiendo esa reflexión etnográfica, espero poner de relieve el papel de un cuerpo altamente especificado -blanco, occi-

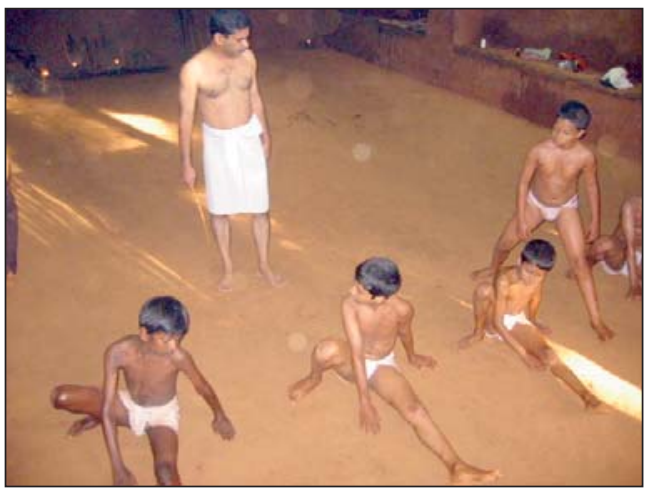
dental, femenino, sin ataduras de marido o hijos- en una situación de aprendizaje intercultural. Las siguientes dos estampas, que ocuparon mi tiempo en el C.V.N Kalari de Calicut, demuestran tanto mi atracción hacia el mundo de la enseñanza y el aprendizaje como mi reconocimiento de sus limitaciones.

\section{Cuándo y qué enseña el profesor a través del tacto} Dos estampas de apoyo

\section{Estampa 1: La bendición del profesor}

Inmediatamente después de entrar en el kalari, el profesor, desnudo de cintura para arriba, comienza a rezar hacia cada una de las plataformas situadas en el contorno de la pequeña y húmeda estructura del suelo y muros de arcilla roja. Su circuito contemplativo, realizado en el sentido de las agujas del reloj, me recuerda a las estaciones del vía crucis católico, empleando cierto tiempo en aparentes oraciones o reflexiones hacia una serie de centros demarcados espacialmente, cada uno de ellos con una o más de las deidades hindús asociadas con las artes marciales. A medida que continúan con sus calentamientos en el kalari cubierto de paja, sus estudiantes de seis a doce años lo miran con una silenciosa atención periférica, con cuidado de no molestarlo en su preparación espiritual para la práctica vespertina.

Pero tan pronto como completa el circuito de las plataformas, los estudiantes más jóvenes se arremolinan a su alrededor. Mientras que cada estudiante toca con sus dedos ligera y rápidamente los pies del profesor y los lleva hacia su propio corazón, repitiendo el gesto varias veces, el gurú deja caer su mano en la coronilla del estudiante, llevándola hacia su corazón y después hacia su cabeza.

Esta bendición habitual de cada estudiante, sin embargo, no acaba con esa conexión gestual y energética entre los corazones y las cabezas del profesor y el estudiante, a través de los pies del gurú, sino con un gesto final de la mano del 
1) Trabajo de suelo para AUMENTAR LA FLEXIBILIDAD.

2) ESTUDIANTES MAYORES PRACTICANDO EJERCICIOS DE PIERNAS.
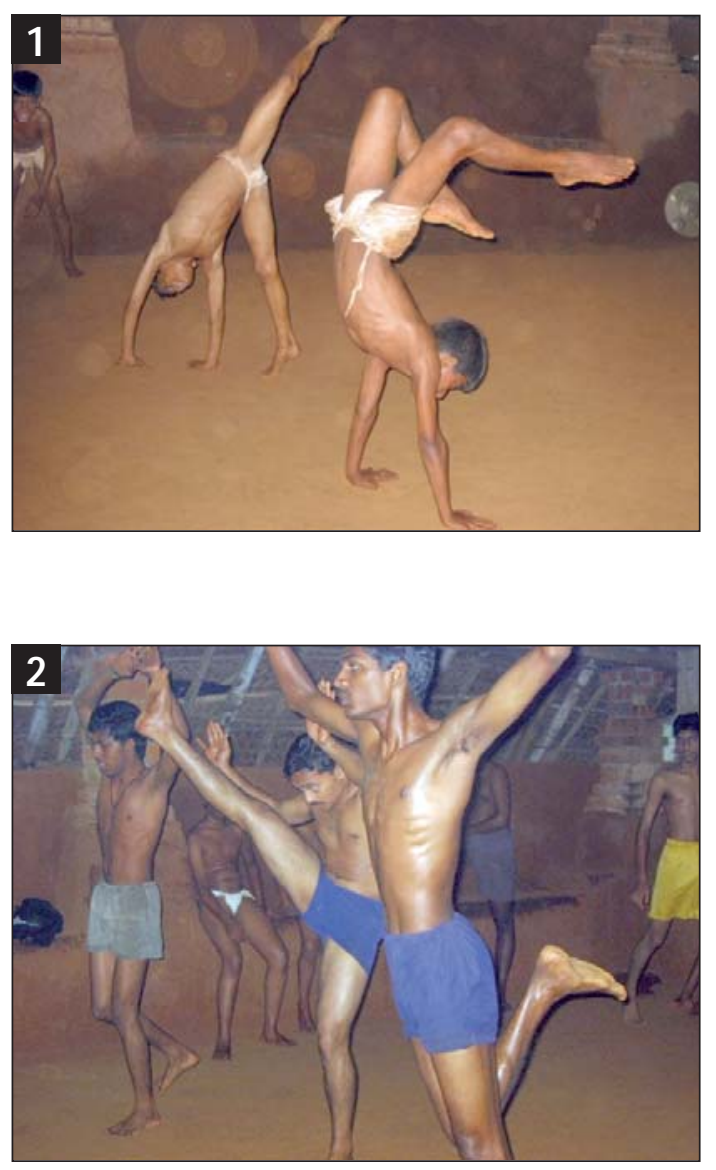

gurú. Desde la coronilla del estudiante y su propia cabeza, el gurú eleva la mano en el espacio, como si conectara tanto la vulnerabilidad del discípulo y su necesidad de ser bendecido como la capacidad del gurú para conferir tal bendición con una fuente mayor de poder y aprendizaje que abraza a ambos.

Como profesora de yoga y universitaria, esta coda gestual de la bendición me llega al alma. En ella vi la sumisión tanto del gurú como del sisya, o discípulo, a una tradición mayor que los envolvía a ambos. Experimento con una profundidad sin precedentes el honor de ser profesora -la humildad y el respeto del estudiante, el amor y fe del profesor y la sumisión de ambos en el regalo del conocimiento y la compresión por las cuales ambos se esfuerzan-.

\section{Estampa 2: La mano negada.}

Algunos meses más tarde me preparo para dejar el C.V.N Kalari, mi gurú y la casa de mis huéspedes, al hermano y la cuñada de mi gurú. Espero que la despedida recuerde la simplicidad y la pureza de la bendición del profesor que me introdujo a la práctica. En vez de eso, los diferenciales de poder financieros y culturales, reales y percibidos, dominan la escena.

Mi profesor me manda liar sobre mi camiseta de práctica, la katcha roja, blanca y negra, una prenda que se enrolla por la cintura y las piernas cuando se realizan muestras de kalarippayattu; su mujer, Anitha, me ayuda. Sudhakaran sin duda sabe que estoy aún a años de práctica de estar preparada para tomar parte en esas representaciones. Sin embargo sé por otras experiencias, tanto con él como en otros lugares durante mi trabajo de campo en la India, que los occidentales con interés duradero en las prácticas culturales indias pueden ser cogidos en sesiones fotográficas por sorpresa cuyo producto puede añadir caché a la institución local. Las fotografías de un occidental en el C.V.N Kalari pueden servir al kalari, así como a la Comisión de Turismo de Kerala, generándose intereses de largo alcance respecto a la idea de que los euro-americanos blancos, al igual que los locales, encuentran interesante entrenarse allí.

Sintiéndome incómodamente como un objeto, trato de manejar la sesión fotográfica intentando producir fotos que pudiera utilizar como referencias para el aprendizaje. Pido al gurú de forma inocente que pose conmigo y haga como si corrige mis posturas -algo que claramente necesita seguir haciendo-. Se detiene un momento, entonces, con vacilación, permite que sus manos toquen ligeramente mis hombros, de un modo que en nada se parece a alguna otra corrección que haya visto durante el entrenamiento, y que es totalmente inefectiva como corrección a la postura que estoy realizando.

De pronto, lo que se estaba gestando en mí a lo largo del periodo de entrenamiento se hace completamente consciente: reconozco que no me ha corregido la postura con la mano ninguna vez: que, a diferencia de lo que ocurre con mi compañero americano, W illiam, que entrenó durante semanas a mi 
lado en el kalari, como mujer he recibido lo que podría considerarse como una ilusión de entrenamiento, ya que Sudhakaran me ha enseñado sobre todo a través de la demostración y la discusión. Por tradición, sin embargo, el kalarippayattu se enseña mediante órdenes verbales en malayalam, golpes rápidos del bastón de entrenamiento sobre los brazos y piernas del estudiante, y a través de ajustes ocasionales posando las manos, tal y como se hace en algunas formas de yoga. Como estudiante de yoga, cuando llegué a la India esperaba recibir correcciones con las manos; mi experiencia dos años antes como estudiante de danza en la Bali hindú me había generado el mismo tipo de expectativas. De hecho, había ido a Indonesia para experimentar cómo sería el entrenamiento de baile cuando el estudiante se comporta como arcilla en las manos del escultor; esperaba contrastar esa experiencia de aprendizaje con la forma de enseñar mediante modelos e instrucción más verbal, tal y como ocurre en ballet, la forma de danza con la que había crecido.

De pronto volví sobre un tópico que el Sudhakaran había sacado, como de la nada, justo después de que fuera aceptada como estudiante (Sudhakaran, comunicación personal, 2002). Había comenzado a hablar sobre relaciones de género en India en comparación a los Estados Unidos y Europa. Es un hecho, comentaba, que hombres y mujeres son diferentes. Más tarde ese día escribí, "No tengo claro cuáles piensa que son esas diferencias. Pero me sorprendí cuando me explicó un poco más sobre el hecho de que cuando aquí un hombre toca a una mujer, siempre es algo sexual; incluso si un hombre y una mujer hablaran en público unas pocas veces, la gente [pensaría] que está pasando algo". Su discurso me dejó realmente confundida: ipor qué siquiera había salido el tema? ¿Había algún subtexto? ¿Trataba de mantener a raya algún tipo de sentimiento hacia mí? ¿Estaba intentando asegurarse de que no lo tocara, como sería costumbre en mi país y mi propio instinto? Entonces me di cuenta de que me estaba explicando por qué W illiam recibiría un tipo de entrenamiento muy distinto al que yo recibiría.

Ya cuando estudiaba en el C.V.N Kalari a mi llegada, W illiam era al mismo tiempo un compañero y una molestia. Era un compatriota estadounidense con el que compartía las mismas observaciones culturales y calamidades durante el entrenamiento. Por un lado, yo estaba feliz por su presencia, ya que ayudaba a dispersar la atención que hubiera recibido como mujer soltera que viaja sola. Sin embargo también notaba la explosión de sentimientos hacia él, que se asemejaban mucho a las rivalidades entre hermanos: estaba continuamente sorprendida por el crédito que Sudhakaran parecía conceder a W illiam -el cual se me antojaba como un compañero desafortunado- preguntándole por ejemplo qué pensaba sobre mi cámara digital de alta calidad.

Al mismo tiempo, a la vez que me invadía el asombro de darme cuenta de la base de género específica que tenía mi aprendizaje, la realización de las fotos tomó en sí una importancia estratosférica. Me di cuenta de que podía ser el más sutil, si bien el menos convencional, de todos los entrenamientos que había recibido en Calicut -aquí, mientras yo ajusto en silencio mi postura, mi profesor aguarda en silencio, el toque correctivo por el cual suspiraba sustituido por su mirada crítica y su silencio hasta que lo haga suficientemente bien-. Intentaba autocorregirme, destilar la esencia de cada movimiento en una sola postura que 
pudiera capturar el espíritu global. Que él permanezca en silencio significa que mis esfuerzos aún son "fríos"; un ligero cambio en su energía podría significar que "me estoy acercando".

La escena pone de relieve la sustitución de un contacto corporal imposible en este ambiente de entrenamiento entre géneros, así como el fundamento de clase que permanece en la interacción entre este objeto de estudio y la trabajadora de campo, entre el gurú y el estudiante, y el profesor masculino del sur de India que pertenece a la histórica casta guerrera y la estudiante femenina de una sociedad estadounidense supuestamente sin clases. Me había sentido tan profundamente conmovida por la sumisión tanto del gurú como del discípulo en el acto de conferir la bendición que había minimizado cualquier dificultad que luego iba a encontrar aceptando mi papel en la obra.

\section{Experimentando un 0 tro experimentándome a Mí como 0 tro.}

El título de la obra de teatro de Caryl Churchill de 1968 cuenta la historia de esto, lo que ocurre en muchos encuentros culturales: era, en cierto modo, The Marriage of Toby's Idea of Angela and Toby's Idea of Angela's Idea of Toby [E] matrimonio de la idea de Toby sobre Ángela y la idea de Toby sobre la idea de Ángela sobre Toby]. Como antropóloga somática estadounidense, estaba abierta tanto a examinar mi experiencia en una nueva cultura como horrorizada por haber entrado en esta sociedad jerárquica con una específica y complicada posición social asignada a mí.

Como puede haber sido evidente para el lector durante algunos instantes, llegué al encuentro con mi propio etnocentrismo bastante intacto. Era primordial sin duda mi profunda convicción estadounidense de igualitarismo, con gente blanca y con gente de color, mujeres y hombres, todos tratados como seres de igual valía. Por tanto, la proyección de las dos caras del pedestal étnico y de género en el que se me había colocado me inquietaba enormemente.

En referencia a "La idea de Toby sobre Ángela", tenía al menos tres deseos enfrentados. Uno, como mencioné previamente, era mantener mi relación de colega con Sudhakaran, en vez de someterme a él como gurú. En un determinado momento del entrenamiento me vi a mí misma llena de historias sobre gurús corruptos en los Estados Unidos, obsesionada con la preocupación (sin ningún fundamento real) de que Sudhakaran pudiera no ser el ejemplo moral que yo "necesitaba" que fuera.

En segundo lugar, había cierta atracción hacia Sudhakaran, un hombre atlético, guapo, cinco años mayor que yo; quizá esto estaba generado en parte por el contacto tanto físico como verbal, prohibidos y llenos de connotaciones. (Por supuesto existe una larga historia de protegidas que proyectan esos sentimientos sobre sus mentores masculinos). Era además bien consciente, durante los dos meses en total que pasé en la India, de tener negado el contacto, tan acostumbrada como estaba a saludar a mis amigos, a compañeros de yoga y ocasionalmente a estudiantes con un abrazo.

Finalmente, estaba la preocupación mayor, común entre los antropólogos, sobre la usurpación de una práctica cultural y la recompensa obtenida sin compartir adecuadamente una parte de los beneficios con la gente a la que pertenece esa 
tradición. Me debatía con ese tema de manera especial porque yo misma financiaba mi investigación allí. ¿Quería realmente ser la única explotadora, exigiendo que en un relativo corto período de tiempo Kerala me entregara sus técnicas y secretos, sólo para irme y hacer algo con ello?

Yendo hacia la parte de "La idea de Toby sobre la idea de Ángela sobre Toby", para las personas con las que trabajé en la India, creo que yo era muchas cosas -alguien que no era ni hindú ni musulmana; una mujer soltera y sin hijos que viajaba sola; una euro-estadounidense de piel clara-. Me sentía extrañamente despersonalizada cuando me enfrenté con categorías de identidad de significados no acostumbrados. Sudhakaran y su familia habían tenido un mínimo o ningún contacto previo con judíos; aquellos que no eran ni hindús ni musulmanes eran por norma cristianos honoríficos. Además, como mujer en India, era alguien cuya piel y figura tenían que ser tapadas en público; alguien -que como Sudhakaran había tratado de hacerme comprender- no podía ser vista hablando sola con, 0 ser tocada por, un hombre sin que los demás asumieran que existía una relación sexual, en esta sociedad de castas declarada. Tenía la esperanza de aprender kalarippayattu en snugs, una especie de top usado en yoga que había traído para la práctica en ambientes calurosos, pero esto me fue inmediatamente prohibido por el gurú, mandándome a comprar camisetas más sencillas. No había lugar a un toque amistoso, entre colegas femeninos y masculinos, con el cual estaba acostumbrada en los Estados Unidos. Tampoco para el tipo atlético femenino tratado de modo abierto y consciente, corporalmente hablando, al que estaba acostumbrada en el mundo del yoga en Chicago. Finalmente, como euro-estadounidense, recibí en la India una proyección duradera como potencial benefactora, tanto financiera como de conexión hacia oportunidades de proyección internacional para los indios.

Frecuentemente, me sentía como si se me hablara con dos códigos contrapuestos: uno que sería utilizado para las personas honoríficas de casta alta y otro que se asociaba con la desconsideración mostrada hacia las mujeres nativas en la India, incluso en el estado relativamente liberal de Kerala. Ninguna de esas proyecciones me resultaba cómoda y yo me esforzaba, medio en broma, con un amigo de Sudhakaran -Prasad, que estaba más a gusto con las costumbres occidentales de lo que estaba mi gurú-, para que me tratara y pensara sobre mí no como una mujer blanca sino jcomo un hombre indio! Dejando a un lado su pragmatismo, conceptualmente esto parecía (naturalmente) ridículo para él -aunque para mí parecía una solución a la sensación incómoda de ser elevada socialmente, tratada como una amenaza sexual permanente 0 ignorada- ${ }^{2}$.

\footnotetext{
2 Y, como alguien nacida judía y viajando a las zonas con más analfabetismo de la India en menos de un año tras el 11 de septiembre de 2001, me sorprendió oír la teoría de que los israelíes y los judíos estadunidenses eran los responsables de los ataques terroristas a las Torres Gemelas de Nueva York, que habían avisado con tiempo a todos los judíos que trabajaban en los edificios para que salieran y no muriesen, ya que de hecho ninguno había muerto. Más tarde aprendí que esa teoría se había expandido por todo el mundo, incluso entre muchos musulmanes y en el Medio Oriente, y que debía considerarse como una repetición en el siglo veintiuno del documento antisemita decimonónico Los Protocolos de los sabios de Sión, que aseguraba que un secreto y poderoso grupo de judíos estaba tratando de manipular los acontecimientos mundiales para servir a los intereses judíos (Anti-Defamation League, 2003, pp. 6-7; N euman, 2005).
} 

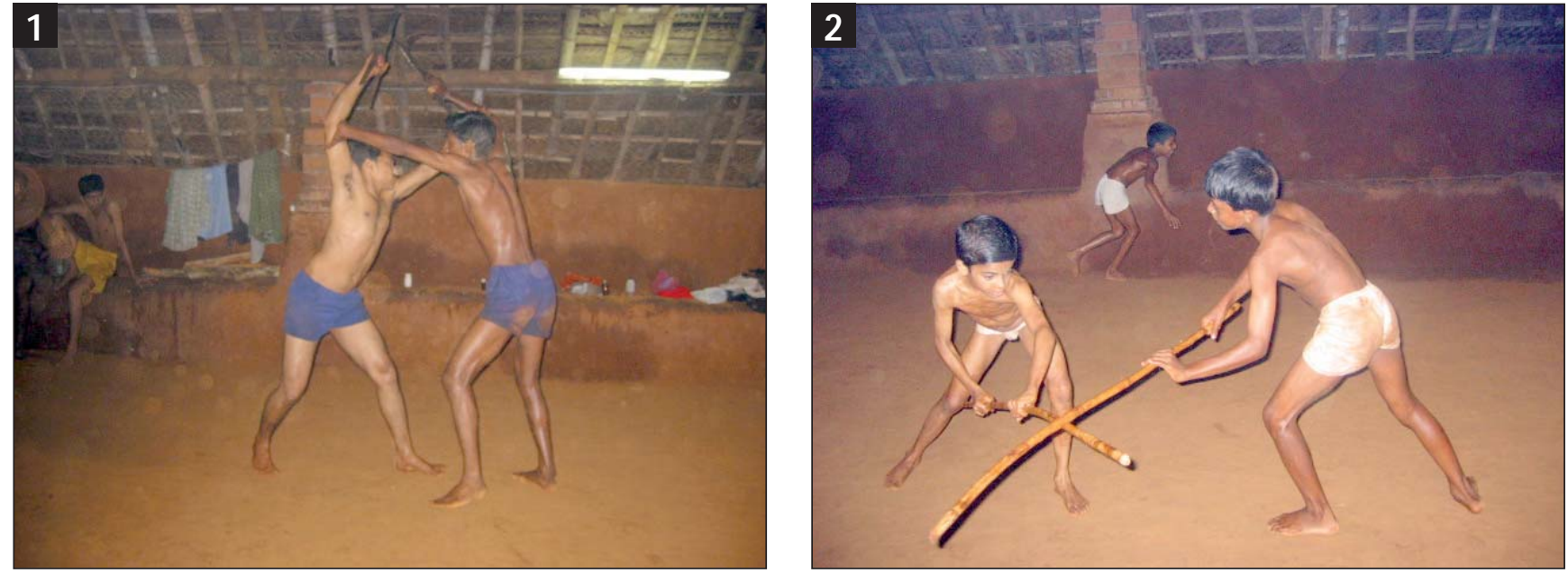

1) Estudiantes mayores practicando la secuencia de palo corto.

2) Practicantes jóvenes practicando con palo largo.
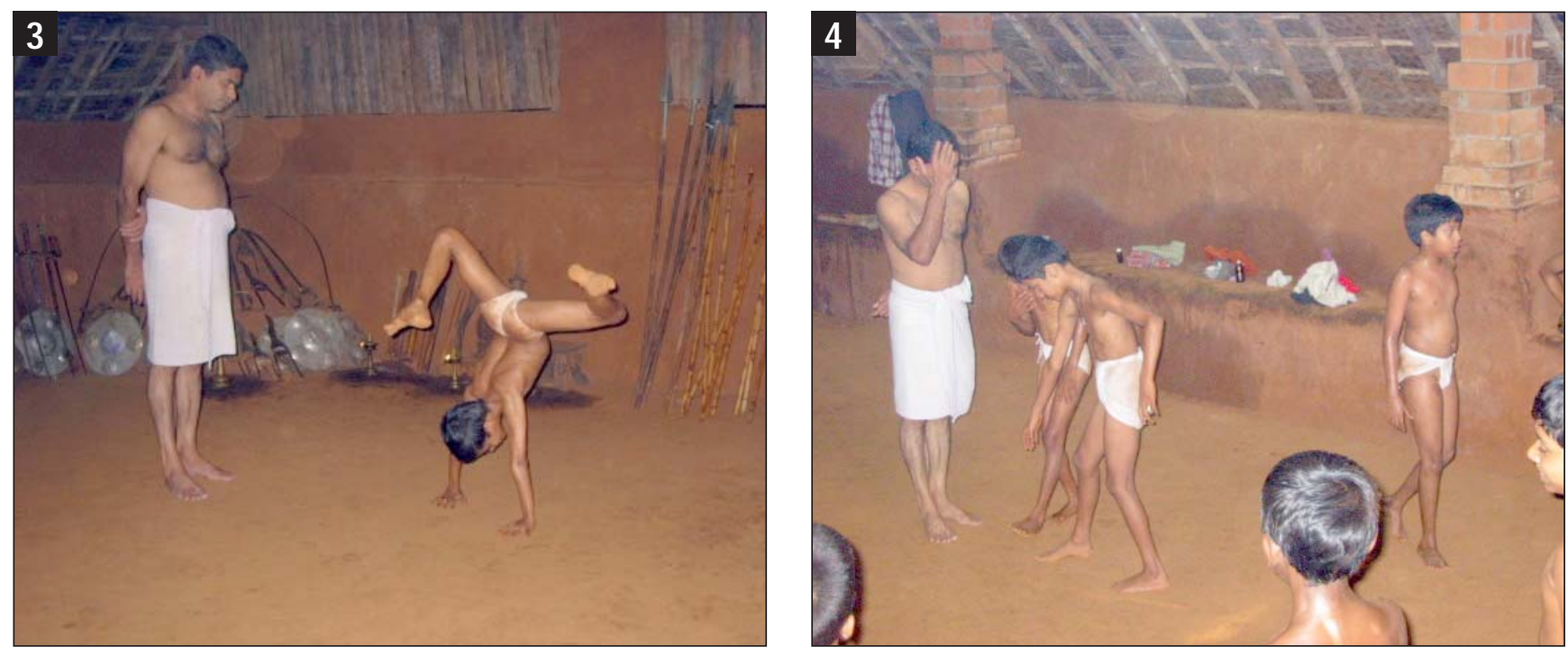

3) Practicando bajo los atentos ojos del profesor.

4) Estudiantes recibiendo la bendición del gurukKal antes de dejar la práctica.

\section{Enseñanza corporal en Kerala}

En el lenguaje de Bourdieu y Shilling, el "cuerpo profesional" es una especie de "capital físico", capaz de transformar el cultivo corporal de años en un modo de comunicación y desarrollo de los demás (citado en Light, 2001). Lave (1977) resaltó la base inductiva del aprendizaje, que se puede aplicar tanto al entrenamien- 
to de las artes marciales orientales en general como de forma específica al kalarippayattu, ya que los estudiantes practican con otros de su misma edad y nivel. Los estudiantes más avanzados participan en el entrenamiento de los menos avanzados, y el gurú sirve como el ejemplo definitivo, no sólo como el que arbitra la práctica. En el kalari de Trivandrum que visité, al sur de Kerala, así como en el C.V.N Kalari en Calicut, los estudiantes practicaban con iguales de su misma edad y nivel, lo cual iba generalmente unido. Así, todos los estudiantes que estaban a mi nivel eran chicos de la mitad de mi tamaño y llevaban taparrabos, o chicas jóvenes en pantalón corto y camiseta, riendo y mirándome a escondidas desde detrás de los arbustos.

En kalarippayattu, los estudiantes son enseñados mediante memorización por repetición. Practican los movimientos a medida que el gurú los nombra y les da correcciones físicas. Los significados subyacentes aparecen con el tiempo. Como Sudhakaran me explicó una vez durante una entrevista, los estudiantes jóvenes sólo reciben el contexto espiritual sobre lo que están haciendo una vez el profesor siente que están preparados (Sudhakaran, comunicación personal, septiembre 2002). Aunque daba a sus estudiantes más jóvenes el esquema básico de las acciones que debían realizar mientras daban vueltas al kalari en adoración, consideraba que el entrenamiento religioso inicial era responsabilidad de los padres. Debido a esto, no entraría de primeras en excesivos detalles sobre el estado mental que debían cultivar mientras rezaban delante de cada una de las deidades situadas en el contorno del kalari: decirle a una persona demasiado pronto que canalice el poder de los dioses en su práctica servirá sólo para distraerlo, en opinión de este gurú.

Una buena parte de la enseñanza en kalarippayattu se hace mediante la verbalización por parte del gurú de los movimientos que deben realizarse al tiempo que se realizan y mediante el contacto, el cual está mediado a menudo por un palo de bambú de una yarda $(0,91 \mathrm{~m})$ que es utilizado como la primera arma de los estudiantes. Por ejemplo, Sudhakaran inclinaría más la parte superior del cuerpo de un estudiante que estaba haciendo sentadilla apoyando el palo en su espalda. Los entrenadores de estudiantes adultos de kalarippayattu en la ciudad del sur de Kerala, Trivandrum, también utilizaban el palo como guía de corrección. Un profesor que observé utilizaba el palo para corregir el giro de la pierna de un estudiante 0 la posición de la parte baja de su espalda, caderas o pecho. Sudhakaran me comentó que los malos profesores podían darse cuenta de los errores de sus estudiantes, pero elegían no corregirles (Sudhakaran, comunicación personal, septiembre 2002).

El castigo corporal a los estudiantes fue prohibido por la Corte Suprema India en el 2000, y la prohibición tan solo ha sido llevada a cabo laxamente. Sin

USANDO UN PALO PARA CORREGIR A UN ESTUDIANTE. 
embargo, en 2002, con estudiantes jóvenes en Kerala, el castigo corporal parecía ser una estrategia disciplinaria y de enseñanza habitual. Vi a Sudhakaran atizar rápidamente a un estudiante que no había puesto en práctica las correcciones previas o no estaba prestando atención. Los chicos en su kalari consideraban que la utilización del palo de bambú allí era mucho menor de lo que sus maestros de escuela hacían cuando se portaban mal en clase (comunicación personal, septiembre 2002). Sin embargo, la hija de Sudhakaran, Archana, me contó que siempre que los occidentales, con su frecuente horror al castigo corporal, venían al C.V.N Kalari, su padre disminuía la intensidad y frecuencia de sus correcciones más duras, así que creía que yo había sido expuesta a un menor grado de corrección corporal de lo que un natural de Kerala hubiera recibido (comunicación personal, septiembre 2002). Los euro-estadunidenses generalmente han tenido desde hace más tiempo prohibiciones respecto al castigo corporal de los estudiantes; N ew Jersey prohibió esa práctica en las escuelas tan tempranamente como en la década de 1860, mientras que en gran parte de Europa continental y de los Estados Unidos la prohibición se ha promulgado en décadas recientes.

Incluso algunas exhortaciones verbales habituales pueden ser cuestionadas por los occidentales. Durante mi estancia en Kerala, visité el Kerala Kalamandalam en Cheruthuruthy, donde se formaba a los chicos, tanto manual como técnicamente, para ser ejecutantes de kathakali, la forma altamente estilizada de danzateatro fuertemente basada en el kalarippayattu (Zarrilli, 2000: 3). El maestro de Kalamandalam, Ramadass, contó la historia sobre la enseñanza de un estudiante holandés que puso en duda su método de instrucción que era gritarle cuando cometía un fallo; argumentaba que, ya que no era de Kerala, ipor qué debía el profesor abusar de él verbalmente al modo de Kerala? Esta interacción, que Ramadass describía como algo profundo para él, le hizo cambiar su comportamiento a la hora de enseñar a estudiantes extranjeros -si bien continuó gritando a los nativos- (Ramadass, comunicación personal, agosto 2002).

Los chicos jóvenes en particular podían ser objeto de un castigo corporal bastante serio. En el Kalamandalam, las narraciones de abusos sobre estudiantes tienen tintes legendarios. Dos chicos adolescentes me contaron que tuvieron que dar cuidados médicos y emocionales a dos chicos más jóvenes que habían sufrido grandes ampollas por las palizas del gurú (Piyal \& Gautam, comunicación personal, septiembre 2002).

En disciplinas físicas con música, tales como el kathakali y la danza clásica india, el gurú suele tener palos que son muy útiles para mantener el tempo en la práctica de los estudiantes. Sin embargo, los palos sirven no sólo para marcar el ritmo. Observé a un profesor de kathakali en el Kalamandalam recorrer una fila de chicos contra la pared de la clase, reprendiendo y golpeando uno por uno. Mientras yo miraba, él se centró en uno de ellos, golpeándole varias veces en la parte posterior de la cabeza, mientras le hablaba mordazmente. Como una reminiscencia de los campos de entrenamiento de reclutas en las películas de Hollywood, el chico de turno aguantaba la mirada estoicamente, nunca protestando abiertamente, mientras el siguiente chico de la línea mantenía su mirada al frente y parecía no inmutarse hasta que el profesor empezaba a hablar con él. 
Las palizas pueden ser no aceptadas y sujetas a cierta respuesta, si bien no está claro cómo de firmes son tales respuestas. Ramadass me contó que fue suspendido tras haber sido acusado (quizá de manera falsa) de haber lesionado a estudiantes mediante palizas. Fue readmitido pero ahora es cauto y dice no haber golpeado a más estudiantes desde entonces (Ramadass, comunicación personal, agosto 2002).

Incluso las estrategias pedagógicas que no son violentas físicamente pueden ser bastante duras emocionalmente. Durante los exámenes finales de kathakali que presencié, cada estudiante mostraba veinte minutos de su material -mucho tiempo para estar sujeto al juicio y observación estrecha de dos profesores, cinco compañeros de clase y una visitante euro- estadounidense-. Como los estudiantes de kalarippayattu, los estudiantes de kathakali en el Kalamandalam deben aprender viendo a los demás. Durante los exámenes, si veía a un estudiante cometiendo un fallo, el profesor, Krishna Kumar, paraba la danza y le preguntaba primero a él, y después a sus compañeros de clase, qué estaba mal en su actuación.

La sala de exámenes se llenaba por completo con la risa burlona del instructor, el ridículo y el refuerzo negativo, tanto físico como verbal. Más tarde, Ramadass me contó que él nunca le dice al estudiante lo que ha hecho bien (comunicación personal, agosto 2002). El estudiante sólo sabe de la impresión de su profesor respecto a él cuando sus padres reciben la tarjeta de informe con un resultado y el ranking que ocupa en su clase.

\section{Conclusión}

En la primera estampa, que tuvo lugar en el C.V.N Kalari de Sudhakaran, yo era una observadora, testigo de una imagen tradicional en Kerala sobre el buscar y ofrecer bendiciones y mostrar respeto entre el gurú y el discípulo, así como por la tradición y la práctica que envolvía a ambos. Vi el contacto y su evanescencia según iba practicándose y realizándose entre los que allí pertenecían. No tenía la sensación de que algo fuera alterado por mi presencia, ni el comportamiento de Sudhakaran hacia sus discípulos ni el de ellos hacia él.

Muchas cosas han cambiado en el momento de la segunda estampa, la cual cerró mi tiempo en el C.V.N Kalari. Yo ya no representaba una cámara distanciada, fotógrafa y entrevistadora que se hospedaba en un hotel de la ciudad. Ahora era una discípula de mi sujeto de investigación, compartiendo casa con la familia de su hermano al otro lado de la calle. Las cualidades de mi propio cuerpo étnico y de género alterarían su práctica -cómo me enseñaría, lo que elegiría para corregir, dónde me colocaría en la práctica-. No había realizado una transición fuera de un rol cuya valencia en la cultura de Kerala era incómoda: era todavía de piel clara, euroestadounidense, mujer, soltera y sin hijos en edad de tenerlos. Cuando tomé la determinación de acercarme más a la práctica del kalarippayattu mediante mi propia inteligencia kinestésica -el modo que consideraba como el mejor camino para mi propia comprensión- paradójicamente limité lo cerca que llegaría o podría llegar a estar del entendimiento que buscaba.

La segunda estampa está plagada de ironías: la relativa superficialidad del contacto en la despedida, a pesar de que habíamos compartido bastante tiempo juntos, la disposición del gurukkal de mostrar una practicante no competente co- 


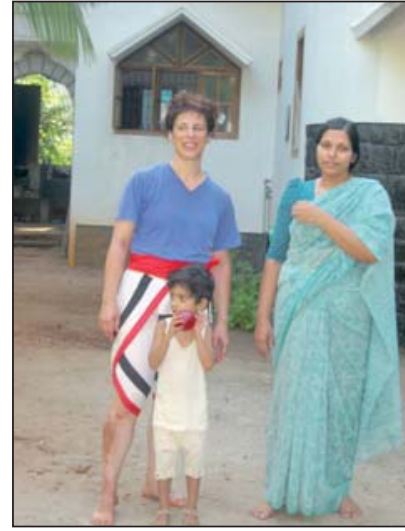

LA AUTORA PORTANDO

UN KATCHA EL ÚLTIMO

DÍA DE ESTUDIO CON SUS ANFITRIONES, LA CUÑADA DEL GURUKKAL Y SU SOBRINA. mo ejecutante del arte por razones de marketing. Quizá, la mayor de las ironías, y el momento que me permitió una mayor comprensión durante mi estancia en Kerala, ocurrió en el momento en que Sudhakaran esperaba en silencio hasta que yo me había corregido suficientemente a mí misma y entonces me reafirmaba con el disparo de la cámara; incorrecta durante el tiempo en que su cámara permanecía muda. Cuando a regañadientes aceptó tocarme en los hombros sin corregirme de verdad, vi la completa ausencia de la enseñanza por el tacto, que yo no me había dado cuenta de que me estaba perdiendo. De algún modo, era como el chico más pequeño que hacía el circuito del kalari en la manifestación exterior del rezo sin entender de forma completa lo que estaba haciendo en cada estación; estaba practicando mi camino hacia la forma con una madurez reducida. Sin embargo, al contrario que ellos, nunca iba a avanzar ya que estaba tratando de aprender sin contar con la forma tradicional del kalari, con la información ofrecida por las normas locales y sociales.

Se ha escrito mucho sobre las diferencias interculturales en comunicación directa e indirecta, así como sobre el uso variado del habla y del silencio, tanto en la enseñanza como en el aprendizaje (véase por ejemplo Radford, 2009; Stahl, 1994; Tincani \& Crozier, 2007). También ha existido cierta atención académica centrada en las diferencias interculturales en el contacto visual y cómo afecta esto a la enseñanza y al aprendizaje (Chiang, 1994; Pitton, W arring, Frank \& Hunter, 1994). Históricamente, en las culturas occidentales, el modo principal en que se ha tratado el tacto como estrategia de enseñanza es, claro está, como castigo físico para disciplinar a alumnos revoltosos. Más recientemente, ciertos autores se quejan por la falta de contacto en las aulas americanas debido a lo políticamente correcto, a una sociedad litigante y a las preocupaciones por la sexualidad emergente de los estudiantes. También ponen énfasis en la presencia de género, sexual de hecho, del profesor (Cooks \& LeBesco, 2006; Freedman \& Holmes, 2003; Johnson, 2006; Sapon-Shevin, 2009).

El tratamiento que realiza Sapon-Shevin (2009) sobre la cuestión de la pérdida sufrida por los alumnos cuando se retira el contacto representa una opinión escasa en su género. Ciertamente, mi propio anhelo del contacto del profesor - magnificado después de darme cuenta de que no lo tuve- tenía muchas fuentes, de las cuales sólo un limitado número podían ser atribuidas específicamente al choque cultural que generé al acudir al entrenamiento de kalarippayattu como mujer adulta esperando el mismo tipo de tratamiento que habría recibido un hombre, tal y como ocurrió en danza y yoga en los Estados Unidos 0, en cierto grado, en el Bali hindú. No obstante, este ejemplo muestra el poder corrector del contacto producido -y negado- y resalta las cualidades de una forma de entrenamiento intercultural en el cual una práctica tradicional descansa en valores tradicionales, incluso en una sociedad globalizante.

\section{REFEREN CIAS}

Anti-Defamation League. (2003). Unraveling anti-Semitic 9/11 conspiracy theories. New York: Gorowitz Institute. Consultado el 20 de abril de 2010 de www.adl.org/anti_semitism/9-11conspiracytheories.pdf 
Behar, R. (1996). The vulnerable observer: Anthropology that breaks your heart. Boston: Beacon Press.

Chiang, L. (1994). Beyond the language: Native Americans' nonverbal communication. (ERIC Document Reproduction Service N O. ED 368540). Consultado de la base de datos ERIC.

Churchill, C. (1968). The marriage of Toby's idea of Angela and Toby's idea of Angela's idea of Toby. Obra teatral no publicada.

Classen, C. (1993). Worlds of sense: Exploring the senses in history and across cultures. New York: Routledge.

Cooks, L., \& LeBesco, K. (2006). Introduction: The pedagogy of the teacher's body. The Review of Education, Pedagogy, and Cultural Studies, 28: 233-238.

Freedman, D., \& Holmes, M. (Eds.) (2003). The teacher's body: Embodiment, authority, and identity in the academy. Albany: State University of New York Press.

Geurts, K. (2002). Culture and the senses: Bodily ways of knowing in an African community. Berkeley: University of California Press.

Johnson, T. (2006). Performing a/sexual teacher: Cartesian duality in education. The Review of Education, Pedagogy, and Cultural Studies, 28: 253266.

Kale, P. (1970). The guru and the professional: The dilemma of the secondary school teacher in Poona, India. Comparative Education Review, 14(3): 371- 376.

Lave, J. (1977). Cognitive consequences of traditional apprenticeship training in West Africa. Anthropology and Education Quarterly, 8(3): 177-180.

Light, R. (2001). The body in the social world and the social world in the body: Applying Bourdieu's work to analyses of physical activity in schools. Consultado el 15 de julio de 2009 de http://www.aare.edu.au/01pap/ lig01450.htm

Ness, S. (1992). Body, movement, and culture: Kinesthetic and visual symbolism in a Philippine community. Philadelphia: University of Pennsylvania Press.

Neuman, J. (2005, 21 de octubre). History of the world, Part 2: Jewish conspiracy theory: The satire. Slate. Consultado el 20 abril de 2010 de http:// www. slate.com/id/2128525/

Osella, C., \& Osella, F. (1998). Friendship and flirting: Micropolitics in Kerala, South India. Journal of the Royal Anthropological Institute, 4(2): 189206.

Pitton, D., W arring, D., Frank, K., \& Hunter, S. (1994). Multicultural messages: Nonverbal behaviors in the classroom. (ERIC Document Reproduction Service N o. ED362519). Consultado de la base de datos ERIC.

Radford, J. (2009, agosto). W ord searches: On the use of verbal and non-verbal resources during classroom talk. Clinical Linguistics and Phonetics, 23(8): 598-610.

Sapon-Shevin, M. (2009). To touch and be touched: The missing discourse of bodies in education. En H. Shapiro (Ed.), Education and hope in troubled 
times: Bold visions of change for our children's world (168-183). N ew York: Routledge.

Stahl, R. (1994). Using "think-time" and "wait-time" skillfully in the classroom (informe de mayo de 1994). (ERIC Document Reproduction Service No. ED30885). Bloomington, IN : ERIC Clearinghouse for Social Studies/Social Science Education.

Tincani, M., \& Crozier, S. (2007, 25 de septiembre). Comparing brief and extended wait-time during small group instruction for children with challenging behavior. Journal of Behavioral Education, 16: 355-367. Consultado de la base de datos ERIC.

Zarrilli, P. (2000). When the body becomes all eyes: Paradigms and practices of power in kalarippayattu, A south Indian martial art. Oxford: Oxford University Press. 